\title{
Planificación docente, en correspondencia con el desarrollo del proceso de Enseñanza-Aprendizaje
}

\author{
Leydi María Tórrez Ruiz ${ }^{1}$
}

\section{RESUMEN}

El propósito del estudio fue analizar la importancia que tiene para el docente de la carrera de Ingeniería Industrial la planificación didáctica y su correspondencia con el desarrollo del proceso de enseñanza - aprendizaje. El diseño Metodológico es cualitativo. Su enfoque se fundamentó en el Paradigma Interpretativo y Socio Crítico, los cuales consisten en comprender e interpretar la realidad educativa desde las concepciones de las personas implicadas en este proceso. Esta investigación según su alcance es exploratoria, trata de conocer la situación referente a la problemática, es descriptiva y explicativa porque se demuestra cómo incide la planificación docente en el desarrollo del proceso de enseñanza y su correspondencia en el mismo y de corte transversal porque se realizó en un período de tiempo determinado. La población de estudio fue el Departamento Ciencia Tecnología y Salud (docentes, directivos y estudiantes). La muestra fueron seis estudiantes que cursan el año, dos directivos (coordinador de la carrera y director del departamento), y seis docentes. Los instrumentos aplicados fueron: entrevista, observación, grupo focal y análisis documental. Para el análisis de datos se realizó la triangulación por fuentes de información. Las conclusiones de la investigación señalan que todos los sujetos, valoran como muy importante la planificación, evidenciándose la correspondencia en algunos documentos de planificación, existiendo discrepancia entre lo que se planifica y lo desarrollado en el aula de clase. Los docentes, estudiantes y directivos expresan la incidencia de la planificación en el proceso de enseñanza-aprendizaje.

Palabras claves: Planificación docente, proceso de Enseñanza-Aprendizaje, importancia, correspondencia, incidencia.

Recibido: 2 de marzo de 2016

Aceptado: 10 de junio de 2016

1 UNAN-Mangua/FAREM-Estelí, Docente horario del Departamento de Ciencia Tecnología y Salud. Correo electrónico: leydimaritorrez@gmail.com 


\title{
Lesson planning, in correspondence with the development of the Teaching-Learning process
}

\begin{abstract}
The purpose of this study was to analyze the importance of lesson planning for teachers of the Industrial Engineering major, and its correspondence with the development of the teaching-learning process. The methodology design was qualitative. Its approach was based on the interpretative and socio-critical paradigm, which aim to understand and interpret the educative reality, from the perceptions of the participants of this process. According to its scope, this is an exploratory research, which intends to know the situation from its problematic, and descriptive-explanatory since it explains the incidence of the lesson planning and its correspondence into the teaching-learning process. Likewise, it is a cross-sectional research since it was carried out in a determined period of time. The population was the Science, Technology and Health Department (teachers, students and directives). The sample consisted of six students, two directives (the program coordinator and the head of the department), and six teachers. The data collection instruments were: interview, observation, focus group and document analysis. For purposes of the data analysis, an information triangulation was made. The main research conclusions reveal that all the research participants value lesson planning as an important element, evidencing the correspondence in some documents for planning. However, a disagreement between what is planned by the teacher and what occurs inside the classroom was found. The teachers, students and directives express the incidence of the lesson planning into the teaching-learning process.
\end{abstract}

Keywords: Lesson planning, Teaching-learning process, Importance, Correspondence, Incidence. 


\section{INTRODUCCIÓN}

La planificación didáctica es un proceso de vital importancia en el quehacer pedagógico del docente, "planear es establecer metas u objetivos por alcanzar, así como definir los pasos por seguir y los medios necesarios para conseguir dichos objetivos". (López Calva, 2008)

El docente independientemente del campo pedagógico que se encuentre debe en todo momento propiciar una reflexión personal del cambio que desea promover en sus estudiantes, un cambio motivado desde él mismo, y para esto necesita planificar pensando en el interés, la necesidad, las expectativas, las características y las limitaciones de sus estudiantes. En la carrera de Ingeniería Industrial existen docentes que tienen conocimientos científicos, pero desconocen algunos aspectos pedagógicos que son esenciales para mediar los conocimientos con sus estudiantes. Ha sido una preocupación de las autoridades universitarias y para dar respuesta a esta necesidad han propiciado espacios de formación pedagogía, a través de Maestrías, diplomados, a aquellos profesionales que desconocen de estos aspectos.

El problema de esta investigación se planteó con la siguiente interrogante: ¿Qué importancia tiene para los docentes su planificación didáctica, y cómo es su correspondencia con el desarrollo del proceso de enseñanza - aprendizaje en la Carrera de Ingeniería Industrial en la FAREM- Estelí, durante el II semestre del año 2015?

Es importante que el docente tenga conocimiento científico y a la vez puedan mediar los conocimientos en el aula de clase optimizando los recursos disponibles para este fin. Esto permite que el estudiante muestre interés por los contenidos y se involucre en las actividades implementadas en este espacio y sea parte de este proceso.
Al respecto Galvis en el año (2012) realizó un estudio titulado "La planificación docente en el proceso de aprendizaje de los alumnos de educación media Colombia", Trabajo de Grado presentado como requisito para optar al título de Magíster en Educación, con mención en Planificación Educativa", cuyo objetivo fue diagnosticar las fortalezas de la planificación docente en el proceso de aprendizaje en la Educación Media. El tipo y diseño de la investigación fue descriptiva y transversal.

Los resultados revelaron:

- La caracterización de los procesos de aprendizaje derivado de la práctica de la planificación docente, que existe en los contenidos de aprendizajes en el área actitudinal un nivel medio alto, en la procedimental un nivel medio y en el área conceptual se encontró un nivel medio bajo.

- Asimismo, al evaluar las operaciones cognitivas, se encontró en un nivel alto la recepción de datos y la retención de la información a largo plazo.

- Continuamos evidenciando un nivel medio en la transferencia del conocimiento y un nivel medio bajo en la comprensión de la información.

Estos resultados permiten dirigir acciones hacia el proceso de aprendizaje de todas las áreas de compromiso, enfocando la atención en aquellas más deficientes. Esta investigación fue relevante para este estudio en cuanto a los fundamentos teóricos de la planificación docente en el proceso de EnseñanzaAprendizaje.

Otro trabajo relacionado con esta temática es el de Renata Rodríguez para optar al título de Doctor en educación y sociedad, titulado "El desarrollo de la práctica reflexiva sobre el quehacer docente, apoyada en el uso de un portafolio digital, en el marco de un programa de formación para académicos de la Universidad Centroamericana de Nicaragua”. Este estudio es una Síntesis sobre la reflexión docente en el que resalta el aspecto de la planificación de la 
enseñanza y la interacción entre profesor y estudiantes como dos elementos fundamentales de la práctica educativa. Esta tesis doctoral sirvió para despertar el interés de investigar la correspondencia que debe tener la planificación con el proceso de enseñanza aprendizaje al igual que su incidencia.

El propósito de la investigación fue analizar la importancia que tiene para el docente del II año de la Carrera de Ingeniería Industrial la planificación didáctica y si está en correspondencia con el desarrollo del proceso de enseñanza - aprendizaje para el desarrollo de su asignatura". Para lograr este propósito se plantearon objetivos específicos y los resultados y conclusiones se realizaron para dar salida a los mismos.

- Conocer la importancia que el docente y los estudiantes de la Carrera de Ingeniería Industrial, le dan a la planificación en el desarrollo de asignatura.

- Interpretar la correspondencia que existe entre los programas de asignatura, el plan didáctico, el plan diario y lo desarrollado en el aula de clase.

- Demostrar la importancia que tiene la planificación didáctica en cada asignatura y su incidencia en el proceso de enseñanza - aprendizaje.

\section{MATERIAL Y MÉTODO}

\section{Diseño}

El diseño metodológico del estudio es cualitativo, pretendió valorar la actividad de planificación de los docentes en la carrera de Ingeniería Industrial y su correspondencia en el desarrollo del proceso de enseñanza-aprendizaje, haciendo uso de datos numéricos que le dan valor agregado a esta investigación sin embargo, el tipo de estudio es cualitativo.

Según Bonilla y García (2002) en la investigación cualitativa el investigador se involucra muy cercanamente con los sujetos de investigación. Existe un interés por conocer el contexto para posibilitar así que el comportamiento de la personas pueda entenderse por dentro del sistema de significados. Así el acceso a la información se realiza a partir de técnicas como la observación participante, las entrevistas, los documentos los registros, etc.

\section{Población}

Para Latorre, del Rincón, y Arnal, otra acción importante del investigador es determinar los sujetos con quienes se va a llevar a cabo el estudio; lo que hace necesario delimitar el ámbito de la investigación definiendo una población.

La población de este estudio fueron los docentes, estudiantes, coordinador de la carrera de Ingeniería Industrial y director, todos pertenecientes al Departamento de Ciencia Tecnología y Salud, en la FAREM- Estelí, UNAN-Managua.

Los autores Latorre, del Rincón, y Arnal se refieren a la muestra como "conjunto de casos extraídos de una población, seleccionados por algún método de muestreo. Se considera muestra grande cuando $\mathrm{n}$ es $=$ 30 o mayor". (Latorre, del Rincón, \& Arnal, 2003)

Los participantes de la investigación fueron seis estudiantes, que cursan el segundo año de la carrera de Ingeniería Industrial. El coordinador de la carrera y director del departamento y cinco docentes activos que imparten clases al año y carrera en cuestión.

Elproceso de selección de la muestra se realizó mediante el muestreo no probabilístico modalidad intencional u opinático ya que todos tenían la posibilidad de ser seleccionados según los criterios de la investigación.

Para Casilimas (2002) este muestreo "tiene su origen en consideraciones de tipo práctico en las cuales se busca obtener la mejor información en el menor tiempo posible, de acuerdo con las circunstancias concretas 
que rodean tanto al investigador como a los sujetos o grupos investigados". (p.124)

Hay que destacar que según Sampieri (2007) "la muestra en el proceso cualitativo, es un grupo de personas, eventos, sucesos, comunidades, etcétera, sobre el cual se habrá de recolectar los datos, sin que necesariamente sea representativo del universo o población que se estudia”. (p. 562)

Por tal razón el número de entrevistado se efectuó por el criterio de la investigadora según su objetivo planteado $\mathrm{y}$ en base a los resultados que pretendía obtener. Al seleccionar a los estudiantes para ser entrevistados se hizo de forma aleatoria siendo único requisito para su selección, pertenecer a la carrera y al año en cuestión.

\section{Entorno}

Este estudio fue realizado en la facultad Regional Multidisciplinaria FAREM-Estelí, UNAN-Managua, ubicada al noroeste de la ciudad de Estelí, en la carrera de Ingeniería Industrial, específicamente en el II año.

\section{Métodos y técnicas}

En una investigación se obtienen cantidad de datos generados por las diferentes técnicas que el investigador utiliza, por tal razón se necesita definir qué métodos y técnicas utilizará en el proceso. Entendiendo por método "conjunto de técnicas y procedimiento que realiza el investigador para la obtención, procesamiento y posterior análisis estadístico de la información....." (López, 2000)

Becerril (1997) "Una técnica de investigación consiste en cómo se realiza la recopilación de la información y cómo se necesita que ésta sea recopilado". (p. 293).

Dado a que el diseño de este trabajo investigativo es cualitativo, los principales métodos señalados por Sampieri para recolectar datos en ésta investigación, son la observación, la entrevista, grupo focal, y la revisión de documentos y materiales. Por tanto, las técnicas utilizadas fueron: guía de entrevista, guía observación estructurada, grupo focal y revisión de documentos.

Los principales aspectos indagados fueron: Importancia de la Planificación, correspondencia que existe entre los programas de asignatura, el plan didáctico, el plan diario y lo desarrollado en el aula de clase y la incidencia en el proceso de Enseñanza - aprendizaje, estas categorías fueron figuradas en cada uno de los instrumentos aplicados a los participantes investigados para conocer su opinión respecto a cada uno de ellos y poder triangular la información y obtener resultados desde los diferentes puntos de vista de los sujetos implicados en el trabajo investigativo.

El método teórico utilizado, fue el inductivo porque se obtuvo información de una muestra seleccionada y los resultados fueron generalizados al expresar las derivaciones de la investigación.

Los métodos empíricos manipulados en este trabajo fueron:

- Técnica de la entrevista, que es la relación directa establecida entre el investigador y su objeto de estudio a través de individuos o grupos. "ésta se define como una reunión para intercambiar información entre una persona u otra". (Sampieri, 2007)

En concordancia con esta definición se diseñó y se aplicó una guía de entrevista estructurada con la intención de que la información requerida no se desviara de los intereses de la investigación de modo que los estudiantes, docentes, y directivos dieran respuestas a las interrogantes.

- Técnica de observación, la observación científica como método consiste en la percepción directa 
del objeto de investigación. La observación investigativa es el instrumento universal del científico. La observación permite conocer la realidad mediante la percepción directa de los objetos y fenómenos.

Para conocer directamente lo que sucede en el proceso de Enseñanza-Aprendizaje se diseñó una guía de observación estructurada no participante, la investigadora se limitó a observar los aspectos que se habían estructurado con anticipación sin desligarse del objetivo de la investigación.

Se observó cómo lo planificado se desarrolla en el proceso de Enseñanza-Aprendizaje en el aula de clase para corroborar lo que el docente $\mathrm{y}$ estudiantes expresaron en la entrevista y en el grupo focal respectivamente.

- Grupo Focal, se realizó un grupo focal donde se diseñó una guía de los temas a tratar con seis estudiantes de la población en estudio, el grupo focal fue dirigido por la investigadora donde se abordaron los mismos aspectos, pero enfocado a estudiantes para conocer su percepción sobre el tema investigado.

El grupo focal es una técnica de uso frecuente posiblemente debido a la facilidad de implementación requiriéndole menos tiempo y en general su aplicación es de menor costo que otras técnicas cualitativas. (López, 2000)

- Análisis de documentos, se analizaron los programas de asignatura, planes didácticos, planes diarios de cada una de las asignaturas que cursan los estudiantes del II año de la carrera en gestión.

\section{Procesamiento y análisis de la información}

Una vez obtenidos los datos, en primera instancia se procedió a realizar la transcripción de los instrumentos aplicados. La información obtenida se clasificó según los objetivos que se plantearon al inicio de la investigación, para los cuales se diseñó cada instrumento. Se redujo la información en matrices por objetivo, facilitando el análisis respectivo para dar salida a los mismos.

Con el objeto de dar más confiabilidad al estudio se procedió a la triangulación, principio que se usa para verificar si los datos obtenidos a través de las diferentes fuentes de información guardan relación o tienen discrepancia entre sí.

En este caso en particular se compararon los datos obtenidos en el trabajo de campo con lo que plantean los autores al respecto y con los antecedentes propuestos en la investigación para dar validez y confiabilidad a los resultados obtenidos y poder redactar las conclusiones y recomendaciones del estudio realizado.

La triangulación según (Cohen y Manion, 1985 y pourtois y desmet, 1988) citado por (Zúniga, 2010) es una de las técnicas más características de la metodología cualitativa. Su principio básico consiste en recoger y analizar datos de distintos ángulos a fin de contrastarlos e interpretarlos.

\section{RESULTADOS Y DISCUSIÓN}

Los principales hallazgos encontrados son presentados por objetivos y las categorías de análisis para lograr los propósitos de la investigación 


\begin{tabular}{|c|c|c|c|c|}
\hline Categorías & Subcategorías & Entrevista a directivos & Entrevista Docentes & Entrevista a estudiantes \\
\hline $\begin{array}{l}\text { Importancia de } \\
\text { la Planificación } \\
\text { docente }\end{array}$ & $\begin{array}{l}\text { Grado de } \\
\text { importancia de } \\
\text { la planificación } \\
\text { docente }\end{array}$ & $\begin{array}{l}\text { Los directivos } \\
\text { entrevistados coinciden } \\
\text { en que la planificación } \\
\text { es de gran importancia, } \\
\text { por ser la guía del } \\
\text { docente en el desarrollo } \\
\text { de clase, mencionando } \\
\text { que el docente se prepara } \\
\text { mejor para impartir los } \\
\text { contenidos. }\end{array}$ & $\begin{array}{l}\text { La mayoría de los } \\
\text { docentes entrevistados } \\
\text { respondieron que } \\
\text { la planificación es } \\
\text { importante porque } \\
\text { guía el proceso de } \\
\text { enseñanza. La minoría } \\
\text { la considera que la } \\
\text { planificación es muy } \\
\text { importante porque } \\
\text { se especifican las } \\
\text { actividades a realizar } \\
\text { con los estudiantes. }\end{array}$ & $\begin{array}{l}\text { El grado de importancia } \\
\text { que los estudiantes } \\
\text { observan en sus docentes } \\
\text { respecto a la planificación } \\
\text { es que es importante, } \\
\text { porque guía el desarrollo } \\
\text { de la clase, la ven como } \\
\text { un proyecto a realizarse } \\
\text { para comprender } \\
\text { mejor los contenidos. } \\
\text { Expresando que de esto } \\
\text { depende el desempeño del } \\
\text { docente. }\end{array}$ \\
\hline
\end{tabular}

\section{Importancia que el docente de la Carrera de Ingeniería Industrial, le da a la planificación para el desarrollo de su asignatura.}

La siguiente matriz reduce la información de todas las entrevistas aplicadas a directivos (coordinador de carrera), docentes que imparten clases al II año de carrera en cuestión y a estudiantes.

\section{Matriz de reducción de datos "importancia que le da el docente a la planificación". Fuente: Elaboración propia}

Hallazgos encontrados: Los docentes directivos y estudiantes coinciden en su mayoría que la planificación es muy importante, y la minoría la considera significativa por las siguientes razones:

- Para el docente es muy importante, porque exige una mejor preparación, además se especifican las actividades a realizar con los estudiantes.

- Los estudiantes han expresado que sus docentes ven con importancia la planificación, porque guía el desarrollo de la clase, la ven como un proyecto a realizarse para comprender mejor los contenidos. Expresan que de esto depende el desempeño del docente.

- Los directivos entrevistados coinciden en que la planificación es de gran importancia, por ser la guía del docente en el desarrollo de clase, destacan que el docente se prepara mejor para impartir los contenidos.

Las consideraciones que los informantes expresan referente a la planificación son las siguientes:

- Para los coordinadores debe ser una de las actividades de la labor docente, evidenciarse en todas las sesiones de clase porque es primordial en su desempeño, considerando los inconvenientes y las actitudes de los estudiantes, concordando con uno de los docentes que enuncia que debe ser una actividad de la labor docente expresa es la parte esencial en la educación. El docente cita que tiene que evidenciarse en todas las sesiones de clase, porque para interactuar con los estudiantes es necesario tener consigo una planificación de los contenidos. Otro la considera que tiene que formar parte en la enseñanza de la asignatura. En este aspecto se encuentran diferentes puntos de vista al considerar la planificación docente.

- Implícitamente los estudiantes consideran que la planificación es parte de la labor docente y evidenciarse en todas las sesiones de clase.

Si la planificación se considera importante y muy importante por los participantes entrevistados, los beneficios que esta tiene para cada uno de ellos son:

- Para los directivos ayuda a determinar acciones en el aula de clase, evitar las improvisaciones que 
puede ejecutar el docente y especificar los fines, objetivos y metas de los contenidos.

- Para los docentes ayuda a organizar las acciones que se realizan en el aula de clase, evitando las improvisaciones $\mathrm{y}$ se especifican objetivos $\mathrm{y}$ metas de los contenidos.

- Para el estudiante ayuda a comprender mejor los contenidos porque el docente se prepara mejor y no improvisa, permitiendo mayor aprendizaje y logro de los objetivos propuestos en la clase.

Para Galván (2008) la importancia de la planificación que realiza el docente radica en que simplifica el trabajo, puesto que constituye en sí misma una guía que permite prever cuales son los resultados de la acción educativa, cómo realizarla y cómo evaluarla. Por tanto la clase que imparte un profesor requiere de una cuidadosa planificación del proceso de Enseñanza-Aprendizaje, que se convierte en condición indispensable para lograr los objetivos deseados. (p.125)

López Calva (2008) expresa que la planificación didáctica ayuda a:

- Aumentar la eficacia de la enseñanza

- Evitar improvisaciones por que confunden al educando.

- Dar secuencia y progresividad a los trabajos escolares

- Proponer las tareas adecuadas de acuerdo con las posibilidades de los estudiantes.

La correspondencia que existe entre los programas de asignatura, el plan didáctico, el plan diario y lo desarrollado en el aula de clase.

En la matriz se figura la estructura que se realizó para el análisis de los documentos de planificación.

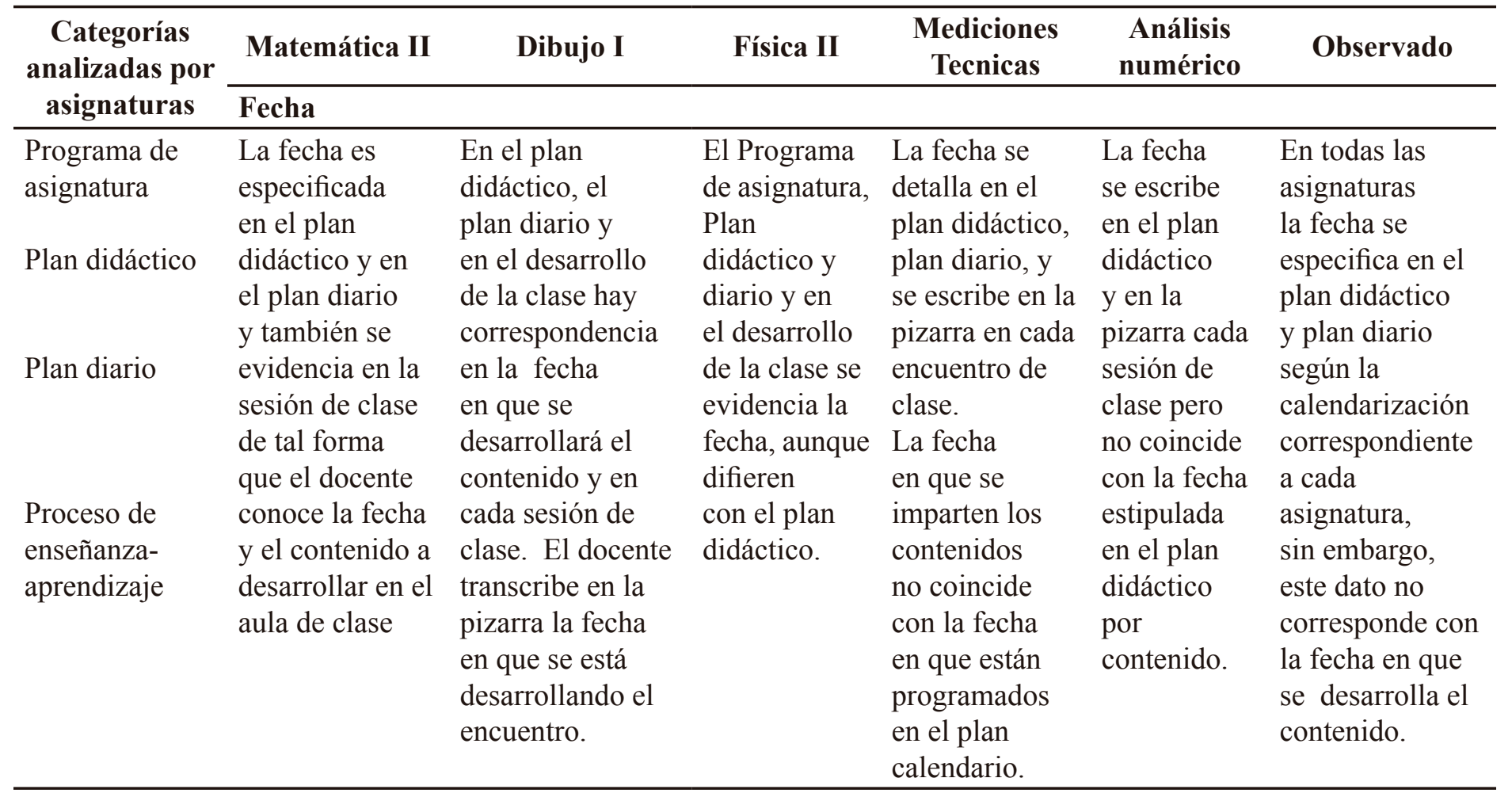

Tabla 2. Matriz de reducción de información de análisis de los documentos de planificación. Fuente: Elaboración propia. 


\section{Hallazgos encontrados:}

- En todas las asignaturas la fecha se especifica en el plan didáctico y plan diario según la calendarización correspondiente a cada asignatura, sin embargo, este dato no corresponde con el desarrollo del contenido en el momento en que se imparten las sesiones de clase con las que se programa.

- En todas las materias el número de sesión de clase se escribe en el plan didáctico pero no en el plan diario.

- En todas disciplinas los objetivos conceptuales, procedimentales y actitudinales están presentes en el programa, plan didáctico y plan diario. Algunos docentes lo dan a conocer al inicio del semestre, otros ni los escriben, ni los mencionan durante o al final del semestre, en las diferentes sesiones de clase.

- En las asignaturas analizadas los contenidos están estructurados por unidades en el programa, en el plan didáctico, en el plan diario y son escritos en la pizarra para ser observados y transcritos por el estudiante.

- En las materias estudiadas los contenidos y subcontenidos estas especificados por unidades temáticas mostrando correspondencia en los documentos de planificación docente y lo desarrollado en el aula de clase.

- En todas las disciplinas el programa sugiere estrategias didácticas de las que se puede auxiliar el docente para impartir la asignatura. En algunos programas existe más diversidad que otros según la naturaleza de la asignatura. Sin embargo cada docente tiene libertad para desarrollar la metodología que considere adecuada en determinado contenido y según la caracterización de los grupos.

- En todas las materias observadas se evidencia la implementación de estrategias didácticas para cada contenido en el plan didáctico, las que en su minoría corresponden a las actividades que el docente planifica en el plan diario y que son utilizadas en la clase. Las estrategias didácticas que más se utilizan son: laboratorios, trabajo cooperativo, indagación de conocimientos previos, trabajos individuales, grupales, exposiciones, discusión grupal, conferencia, guía de lectura y guía de ejercicios.

- En todas las asignaturas el docente planifica las actividades a realizar en su clase asignándoles tiempo para su realización, concretando que dentro de estas actividades no se planifican actividades motivacionales que ayuden al estudiante a despertar el interés por la asignatura y a sentirse motivado en su proceso de aprendizaje.

- En todas las materias los programas y planes hacen mención de las estrategias que debe hacer uso el docente para evaluar la clase según la naturaleza de la clase.

- En todos los planes didácticos de las asignaturas consultadas hacen referencia a las estrategias de evaluación para los contenidos.

- En ambos documentos las estrategias se refieren a la evaluación sumativa para lograr el porcentaje de acumulado, no a otras estrategias de evaluación que permitan mejorar el desarrollo de la clase como la autoevaluación, coevaluación, para retroalimentación entre estudiantes y docentes para mejoras en el proceso de enseñanza-aprendizaje.

- Solo un programa hace referencia a estas estrategias de evaluación.

- El programa, plan didáctico y plan diario en todas las asignaturas especifican los recursos a utilizar en el aula de clase, sugiriendo con más frecuencia en cada una de ellas.

- Todos los docentes hacen uso de recursos tecnológicos como el data y la computadora y recursos convencionales como: (Pizarras acrílica, marcadores, borradores) textos impresos pero en su minoría los docentes utilizan otros como Videos, imágenes que ayudan a los estudiantes a comprender mejor alguno de los contenidos, como instrumentos de laboratorio y de mediciones.

- Hay que tratar de hacer uso de recursos diferenciados y de acorde con el contenido, de tal forma que permita mejor comprensión de los mismos. 
- En todas las materias el tipo de evaluación que más se maneja es la sumativa tanto en el programa, plan didáctico y diario.

- En el desarrollo de la clase no se evidencia el tipo de evaluación que ejecutará el docente para cada contenido, siendo desconocido para el estudiante. La evaluación más utilizada es la sumativa y la diagnostica. Entre la sumativa se aplica guías de ejercicios de forma grupal, pruebas sistemáticas, exámenes, exposiciones e investigaciones.

- El programa menciona algunas estrategias pero difieren en lo planificado y la forma en que se evalúa.

- La mayoría de los docentes planifica y orienta tarea para el próximo encuentro, pero no todos evidencian en el siguiente encuentro el desarrollo de la misma.

- En todas las clases el programa recomienda bibliografía pero no se evidencia en la planificación diaria, indicando algunos estudiantes consultados en la observación que al inicio de la clase el docente les da a conocer la bibliografía que pueden consultar para todos los contenidos a ser abordados.
Existe correspondencia en la mayoría de los elementos entre el programa de asignatura y plan didáctico, encontrando discrepancia en el plan diario y lo implementado en la clase con respecto a los anteriores, es decir que corresponde el programa de asignatura con el plan didáctico, la diferencia radica en algunos elementos del plan diario y con lo que se desarrolla en el 'proceso de enseñanza-aprendizaje.

Medina Rivilla y Mata (2002) expresa que una de las notas más características de una planificación didáctica, puede concretarse en la coherencia, cuando la planificación forma parte de un diseño planificador más amplio con el que guarda relaciones mutuas de dependencia e información. Proyecto del centro y planificación del aula se implican mutuamente en un proceso educativo continuo.

Al respecto Galván (2008) define al programa institucional como un instrumento que facilita el logro de aprendizajes valiosos, es el punto de partida para la planificación docente. La planificación consiste en una estructura sistemática y coherente de los elementos que intervienen en el proceso de enseñanza-aprendizaje.

\section{Incidencia de la planificación didáctica en cada asignatura en el proceso de enseñanza-aprendizaje.}

\begin{tabular}{|c|c|c|c|c|c|}
\hline Categorías & Subcategorías & $\begin{array}{c}\text { Entrevista a } \\
\text { directivos }\end{array}$ & $\begin{array}{c}\text { Grupo focal } \\
\text { con estudiantes }\end{array}$ & $\begin{array}{c}\text { Entrevista a } \\
\text { docentes }\end{array}$ & Consolidado \\
\hline $\begin{array}{l}\text { Incidencia } \\
\text { de la } \\
\text { planificación } \\
\text { docente. }\end{array}$ & $\begin{array}{l}\text { Planificación } \\
\text { facilita el } \\
\text { proceso de } \\
\text { enseñanza } \\
\text { aprendizaje. }\end{array}$ & $\begin{array}{l}\text { Todos convienen } \\
\text { en que la } \\
\text { planificación } \\
\text { facilita el proceso } \\
\text { de enseñanza } \\
\text { aprendizaje, dado } \\
\text { a que el docente } \\
\text { se prepara bien } \\
\text { científicamente, } \\
\text { es una guía en } \\
\text { la clase por } \\
\text { consiguiente } \\
\text { facilita un mejor } \\
\text { desempeño del } \\
\text { docente en el } \\
\text { aula de clase. }\end{array}$ & $\begin{array}{l}\text { Todos los } \\
\text { participantes } \\
\text { investigados } \\
\text { consideran que } \\
\text { la planificación } \\
\text { del docente } \\
\text { facilita su } \\
\text { aprendizaje } \\
\text { porque se trata } \\
\text { con claridad lo } \\
\text { estudiado en el } \\
\text { aula de clase. }\end{array}$ & $\begin{array}{l}\text { Los } \\
\text { entrevistados } \\
\text { enuncian que la } \\
\text { planificación de } \\
\text { los contenidos } \\
\text { facilita el } \\
\text { proceso de } \\
\text { enseñanza } \\
\text { porque en ella se } \\
\text { especifican las } \\
\text { actividades, sirve } \\
\text { de guía, y facilita } \\
\text { el desempeño } \\
\text { del docente y el } \\
\text { aprendizaje del } \\
\text { estudiante. }\end{array}$ & $\begin{array}{l}\text { Todos los sujetos indagados } \\
\text { respondieron que la } \\
\text { planificación facilita el proceso } \\
\text { de enseñanza aprendizaje por } \\
\text { las razones siguientes: } \\
\text { - El docente se prepara bien } \\
\text { científicamente, es una guía } \\
\text { en la clase por consiguiente } \\
\text { facilita un mejor desempeño } \\
\text { del docente en el aula de clase. } \\
\text { - Se trata con claridad lo } \\
\text { estudiado en el aula de clase } \\
\text { - En ella se especifican las } \\
\text { actividades, sirve de guía, } \\
\text { y facilita el desempeño del } \\
\text { docente y el aprendizaje del } \\
\text { estudiante. }\end{array}$ \\
\hline
\end{tabular}




\section{Hallazgos encontrados}

En la tabla se muestra los resultados de las entrevistas a docentes, directivos y el grupo focal con estudiantes sobre la incidencia que tiene la planificación docente en el proceso de enseñanza aprendizaje.

Los docentes, estudiantes y directivos respectivamente han comentado que la planificación si incide en la enseñanza y en el aprendizaje por las siguientes razones:

- Porque el docente se prepara bien científicamente, es una guía en la clase por consiguiente facilita un mejor desempeño del docente en el aula de clase.

- Se trata con claridad lo estudiado en la clase

- En ella se especifican las actividades, sirve de guía, y facilita el desempeño del docente y el aprendizaje del estudiante.

Otro aspecto que se cuestionó a los participantes del estudio fueron las ventajas que les ocasiona la planificación y al respecto contestaron:

Los docentes en su mayoría expresaron que guía el desarrollo de la clase, se definen los objetivos, las actividades, los recursos didácticos, se plantean las estrategias de aprendizaje y se estipula la evaluación del contenido desarrollado, pero la minoría de ellos respondió que la única ventaja que le ocasiona es diseñar estrategias para lograr la comprensión de los contenidos en sus estudiantes, esto indica que no todos los docentes ven a la planificación como algo que incide en el proceso de aprendizaje. Al igual los directivos expresaron las mismas ventajas que los docentes, coincidiendo en este aspecto.

Al respecto los estudiantes expresaron que es una guía para el desarrollo de los contenidos, se especifican con claridad los objetivos y permite medir el logro de estos al finalizar la signatura si hubo aprendizaje o no.
El docente define claramente las actividades que se desarrollan en la clase especificando el tiempo para cada una de ellas. La planificación permite que el docente estipule la forma de evaluar la clase y los recursos para facilitar el proceso.

En definitiva la planificación tiene mucho que incidir en el proceso de enseñanza-aprendizaje creando muchas ventajas tanto para los docentes como para los estudiantes, en ambos se logran las metas propuestas cuando el docente logra efectuar una planificación de acorde a los intereses de los estudiantes y de las exigencias de la sociedad ajustándose a los recursos y habilidades y destrezas de los estudiantes y a los fines de cada asignatura.

\section{CONCLUSIONES}

\section{Importancia que el docente de la Carrera de Ingeniería Industrial, le da a la planificación para el desarrollo de su asignatura.}

Los resultados obtenidos permiten concluir que los docentes expresan que la planificación didáctica es muy importante en el desarrollo del proceso de EnseñanzaAprendizaje al igual que para los estudiantes y directivos que trabajan con la carrera de Ingeniería Industrial.

Los docentes tienen percepciones diferentes en la forma de evidenciar la planificación, unos expresan que debe visualizarse en todas las sesiones, otros en cambio especifican que es una de las actividades propias del docente, en su minoría, que ésta forma parte en la enseñanza de la asignatura. Cuando la planificación debe percibirse de forma integral en todas las sesiones de clase, por obligación y conciencia es una de las labores esenciales del docente comprometido con la enseñanza y estas percepciones no se pueden ver por separadas. 
Se evidencia que los docentes cumplen con su planificación al impartir la clase, pero no incluyen aspectos o acciones importantes para que sea eficaz. Una de las acciones que los docentes mencionan, es que utilizan el análisis del programa de la asignatura, pero otra acción importante que se debe efectuar en la planificación es analizar y ajustar los objetivos a las características de sus estudiantes, acción que no fue mencionada por los docentes consultados, por consiguiente los discentes han expresado que en ciertas ocasiones se escucha que "la clase es aburrida", o que "terminó la asignatura y no aprendieron nada".

Cuando el docente no incluye en su planificación la utilización de medios didácticos que le facilite el aprendizaje en los estudiantes y utiliza los mismos medios o estrategia ocasiona aburrimiento y desinterés en el discente.

Lo que se menciona anteriormente se le atribuye a que el docente muchas veces desconoce algunos aspectos que debe incluir en su planificación aunque cumpla con un esquema que responda a las interrogantes que son requisito para su realización, pero hay que estudiar en lo posible la población estudiantil y el perfil de la carrera, y dinamizar las sesiones de clase, que despierten el interés de los estudiantes en el aprendizaje.

Este efecto se le adiciona a que los docentes poseen conocimiento científico, y desconocen algunos aspectos pedagógicos necesarios para desempeñar la docencia.

La correspondencia que existe entre los programas de asignatura, el plan didáctico, el plan diario y lo desarrollado en el aula de clase.

Referente a esto se puede expresar:

- Que existe correspondencia en la mayoría de los elementos que forman parte de cada uno de los escritos y que son esenciales en la planificación entre estos tenemos: las unidades con sus contenidos y subcontenidos de cada asignatura, los objetivos conceptuales, procedimentales y actitudinales, la bibliografía, las tareas, estrategias de enseñanzaaprendizaje, estrategias de evaluación, todos estos elementos corresponden en el programa de asignatura, el plan didáctico y el plan diario.

- Existe discrepancia en algunos aspectos que son planificados en el plan didáctico y el plan diario, con lo desarrollado en el aula de clase al respecto se puede mencionar que la fecha en que se planifica desarrollar un contenido en el plan didáctico no corresponde con la fecha en que se desarrolla observando un desfase de hasta tres sesiones de clase.

- Otro aspecto que no corresponde son las estrategias de Enseñanza-Aprendizaje y de evaluación descritas en el plan didáctico y lo planificado con lo que se desarrolla en la sesión de clase y lo que propone el programa de la asignatura, aunque hay que destacar que la planificación debe ser flexible a cambios siempre y cuando colabore con el aprendizaje de los estudiantes.

- El programa sugiere estrategias didácticas de las que se puede auxiliar el docente para impartir la asignatura. En algunos programas existe más diversidad que otros según la naturaleza de la asignatura.

- Las estrategias de evaluación que se observó que más utiliza el docente son: el trabajo grupal exposiciones, laboratorio, resolución de guías de ejercicios.

En el análisis de documentos involucrados en la planificación (programa de asignatura, plan didáctico y plan diario) y lo observado en las sesiones de clase se puede expresar:

- Relacionando los resultados de este objetivo con los del primero, se deduce que al no corresponder la fecha en que se planifican los contenidos con la fecha en que se desarrollan, ocasiona que al 
final de la asignatura no se logre desarrollar todo el programa propuesto y es aquí donde los docentes le dan al estudiante la tarea de investigar ciertos contenidos que son de gran importancia y que deben ser desarrollados con cierto grado de profundidad como se evidencia en la matriz del primer objetivo o simplemente el estudiante expresa que no aprendió nada, teniendo dificultad en las asignaturas que están relacionadas a estas.

- No se observa la implementación de actividades motivacionales en ningún documento de la planificación ni en lo observado en la clase y los medios didácticos que se estipulan en el plan diario en correspondencia en algunos casos con el programa son los mismos en casi todas las sesiones de clase al igual que las estrategias de enseñanzaaprendizaje, lo que ocasiona aburrimiento en los estudiantes y desinterés por la asignatura.

- La bibliografía solo se evidencia en el plan programa de asignatura pero no se encuentran plasmadas en el plan diario la bibliografía utilizada por cada contenido.

- Los objetivos son expuestos por el docente a los estudiantes al inicio de la asignatura y algunos de ellos solo hacen mención de los objetivos conceptuales, lo que no permite medir los resultados con los objetivos propuestos para ser evaluados y retroalimentarse para mejoras en el proceso, esto se evidencia en que no se utiliza otro tipo de evaluación más que la sumativa para asignar una calificación.

\section{Incidencia que tiene la planificación didáctica en cada asignatura en el proceso de enseñanza - aprendizaje}

Se identificó claramente las razones por las que incide la planificación didáctica en el proceso de enseñanzaaprendizaje. Estas son:

- El docente se prepara bien científicamente,

- Guía el desarrollo de la clase
- Se trata con claridad lo estudiado en el aula de clase

- Se especifican las actividades y facilita el desempeño del docente y el aprendizaje del estudiante.

- Se definen los objetivos de la clase, los recursos didácticos y se plantean las estrategias de aprendizaje y de evaluación.

La incidencia de la planificación abarca el desempeño profesional del docente y en el aprendizaje de los estudiantes.

La planificación merece una especial atención por el docente para no entorpecer el proceso de enseñanzaaprendizaje y lograr los objetivos propuestos en la asignatura y cumplir con las expectativas de los estudiantes.

Las acciones que debe realizar el docente son: planificar, realizar y evaluar, donde las últimas dos están en dependencia de la planificación que se efectué.

"Se asume que ser buen profesor es saber enseñar bien: dominar los contenidos de la disciplina y saber explicarlos claramente. Si los alumnos aprenden o no depende de muchas otras variables (motivación, capacidad, tiempo dedicado al estudio, estrategias de aprendizaje) que quedan fuera del control de los docentes.....cuando hablamos de enseñanza nos referimos también al proceso de aprendizaje: enseñar es gestionar el proceso completo de enseñanzaaprendizaje que se desarrolla en un contexto determinado, sobre unos contenidos concretos y con un grupo de alumnos con características particulares" (Zabalza, 2007)

\section{BIBLIOGRAFÍA}

Arredondo Galván, V. M. (2008). Didactica General. México: Limusa .

Becerril, f. R. (1997). Ciencia, metodología e investigación. Naucalpan de Juárez: Longman de México Editores S.A. 
Casilimas, C. A. (2002). Investigación Cualitativa. Bogotá Colombia: ARFO Editores e Impresores Ltda.

Galván, V. A. (2008). Didáctica General. Balderas México : Limusa.

Galvis, M. S. (2012). La planificación Docente en el proceso de Enseñanza Aprendizaje de los alumnos de educación media de Colombia. Maracaibo: Universidad de Zulia.

Latorre, A., del Rincón, D., \& Arnal, J. (2003). Bases Metodológicas de la Investigación Educativa. Ediciones Experiencias S.L.

López Calva, M. (2008). Planeación y Evaluación del proceso enseñanza-aprendizaje. México D.F: Trillas.

López, J. P. (2000). Introducción a la Metodología de la Investigación Científica. Managua.

Medina Rivilla, A., \& Mata, F. S. (2002). Didáctica General. Madrid: Pearson Educación.

Sampieri, R. H. (2007). Metodología de la Investigación. México: Ultra.

Zabalza, M. Á. (2007). La enseñanza universitaria. Madrid: Torrejón de Ardoz.

Zúniga, F. J. (2010). Desarrollo de los procesos familiares ante el conocimiento de la orientación sexual de los hijos. Estelí. 